\title{
CARDINAL WTRL: TECHNOLOGY MATURITY, SCHEDULE SLIPPAGE AND TREND FORECASTING.
}

\author{
Behdinan, Kamran; \\ Mishra, Soumya Ranjan \\ University of Toronto, Canada.
}

\begin{abstract}
Maturity assessments of technology is a crucial process to identify and acquire compatible technologies for a system's development. However, being a complex and highly subjective process, the Government Accountability Office (GAO) has reported cost overruns and schedule slippages through the years. This study provides a unique Weighted Technology Readiness Level (WTRL) framework which utilizes cardinal factors to ascertain the maturity, schedule and trend of NASA's 7 Technologies based on their maturity time. The framework utilizes MCDM methods to determine the cardinal complexity of each TRL. It allows the assimilation of other cardinal factors using a simple, open structure to track the overall technology maturity and readiness. Furthermore, this study highlights the importance of tailored TRL frameworks to determine the accurate cardinal coefficient of the said technology and the inferences derived otherwise. It eliminates several limitations of previous frameworks and compares against their performance using a verified statistical representation of processed data.
\end{abstract}

Keywords: Risk management, Technology, Design management, Systems Engineering (SE)

\section{Contact:}

Mishra, Soumya Ranjan

University of Toronto

MIE

Canada

sr.mishra@mail.utoronto.ca

Cite this article: Behdinan, K., Mishra, S. R. (2021) 'Cardinal WTRL: Technology Maturity, Schedule Slippage and Trend Forecasting.', in Proceedings of the International Conference on Engineering Design (ICED21), Gothenburg, Sweden, 16-20 August 2021. DOI:10.1017/pds.2021.60 


\section{INTRODUCTION}

Designing a good system or product requires the innovative culmination of technology and ideas to satiate consumer needs. In large systems, the formulation of a good design is often the foundation to synergise multi-disciplinary interactions. The process of inception can be strongly accelerated through the acquisition and absorption of pre-existing technologies into the said design. One of the major challenges in design can be attributed to transferring a sufficiently sophisticated developed technology across disciplines. Several techniques have been implemented by industries to ensure reliable design preparation in early stages of development. Accurate strategizing of resources and key development stages is crucial to track the product to reach its intended customers. In this study, a numerically computational framework is introduced based on NASA's Technology Readiness framework. The introduced framework is intended to statistically strategize resource allocation while accounting for the development complexity at each stage of development. This is carried out while tracking the degree of sophistication or the maturity of the technology developed.

Assessing the maturity of a defined technology requires assessing its readiness to function in a number of conditions with the eventual goal of transferring it to the customer. The Technology Readiness Level (TRL) developed by NASA was initially aimed at the efficient operation of its space projects and internalised operations (Mankins, 1995; Blanke, 2010). In the years that proceeded, the framework was further projected on numerous other sectors and forums, such as the Department of Defence (ASD(R\&E), 2011) and the Department of Energy (DOE, 2011) in U.S., the European Union (Mihaly, 2017) and many other foreign organisations. Compliance of an acquisition programme also requires evaluating the fitness of a new technology to suit the system's criteria and the expected operational result.

As stated by the Government Accountability Office in U.S. (GAO) (2008) over the years, the Department of Defence (DoD) acquisition projects are facing difficulties with regard to schedule slips, cancellations and failure to achieve efficiency expectations. This was due to insufficiently sophisticated equipment, inadequate architecture and lack of technology maturity. The GAO also proposed some of the best practises for optimising the evaluation and procurement of justified technology for the effective design of the overall system (1999). Technology maturity assessment is a well-established approach utilised for engineers and programme managers to make critical decisions upon the technology's ability to contribute towards system performance. As a consequence, a variety of approaches have been developed for the demarcation of technical growth risks by numerous researchers over the years (Valerdi, 2004; Mankins, 2009).

Cornford et al. (2004) argue that while TRL provides a high-level understanding of technical complexity, it lacks accuracy and precision. There is a need for a thorough concept of technology maturity to allow strategic decisions at critical programme junctures to minimise cost overruns and schedule delays. Similarly, the research undertaken by Olechowski et al. (2020) has illustrated the numerous limitations of the TRL model being used for the evaluation of readiness. In 2009, Azinian et al. carried out a systematic analysis of the numerous types of TRL used in different fields and grouped them into three categories, namely qualitative, quantitative and automatic. The study established the need to create a hybrid architecture with quantitative and automated processes for enhanced maturity measurement. In order to meet this need, Fahimian and Behdinan (2015) presented a framework for calculating the cardinal coefficients of 7 NASA Technologies using the maturation period data made accessible by Piesen et al. (1999). The former framework was further extended towards the determination of Design Readiness Level (DRL) by Revfi et al. (2020). The DRL later on presented by Behdinan (2020) is an emerging method which coalesces the influence of numerous correlated processes to determine the operational maturity of a technology. This framework was later on utilized towards defining the principles of "Axis of Design".

The study by Fahimian and Behdinan (2015) found contradictions in the Weighted Technology Readiness Level (WTRL) approach used due to its ordinality (Lee, 2003) and indicated a quantitative need for maturity assessment (Dubos, 2008; Magnaye, 2010). The WTRL method utilizes the assignation of weights to specific TRL in order to assimilate the complicacies of the development process. These weights are then used as a product of each assigned TRL. However, it is important to understand that TRL in its original state are strictly ordinal, i.e., we can use alphanumeric characters to describe each TRL and still retain the meaningfulness of data assigned to it. It has used the novel 
cardinal system developed by Conrow (2011) to assess the maturity of these technologies. However, there were some flaws in the framework, which are further addressed in the following sections.

\section{RESEARCH OBJECTIVE}

Driven by the framework proposed by Fahimian and Behdinan (2015), Revfi et al. (2020) and Behdinan (2020), this paper formulates an enhanced and novel approach to maturity evaluation. In addition, it assesses and correlates the maturity assessment output of the 7 NASA technologies documented by Piesen et al. (1999). It also demonstrates the need for a targeted TRL framework for each technology to help evaluate the maturity of the technology and recognise projected risks to the system.

The framework aims to remove all previous restrictions as proposed by Fahimian et al. (2015), to provide consistent entry and exit points for the maturity assessment process. Also, it overcomes the major restriction of assigning a "0" time interval to the stages while mathematically satisfying the maturity computation. It is utilized to evaluate the local and global maturity of technology at each TRL and ensure its scalable to use real-time data for efficient preparation and tracking of a technology development process. This paper also provides basic examples of assessing schedule slippage and resource allocation forecasting which could be applied to the mathematical model within the proposed framework.

Technology acquisition for system development is an important process. In order to make key decisions and easy assimilation of the technology into the system one must consider a large number of parameters for assessment. Parameters, often subjective, are difficult to interpret and may not be accurately implemented in mathematical formulations. The model discussed in this paper is intended to be applied towards the active assessment of technology and easy interpretation of subjective end point sub-processes such as quality for technology formulation and acquisition.

\section{ANALYTICAL METHOD FOR WTRL FRAMEWORK}

The cardinality of the TRL system plays a crucial role in the consistency and functioning of the maturity appraisal computational model i.e., if a numerical computational model utilizes an ordinal framework it will yield inaccurate results as one cannot perform mathematical functions on the said TRL itself. Also, for an accurate interpretation of maturity, the cardinal values shall be carefully calculated in order to discern the degree of complexity with each successive level of technology readiness. Conrow (2011) claimed that the original structure developed by NASA was strictly ordinal and used subjective parameters for the assessment of technology maturity and the transfer of technology to a certain degree of readiness. It therefore used the Multi-Criteria Decision Making (MCDM) approach known as the Analytical Hierarchy Process (AHP) developed by Saaty (1990) to evaluate the cardinal coefficients for the appropriate TRL. The approach utilises pairwise comparisons to evaluate the hierarchy of parameters based on the professional judgement of their respective technologies. The system allows users to assess the weights of arbitrary criteria dependent on human feedback. Applying this approach in the sense of the TRL, it was suggested that a jury of experts could attribute the complexity/difficulty of each stage of technical readiness on a scale of 1 to 9 to assess the weights of each level of readiness. These weights, when normalized, would shape the cardinal coefficients of the TRL on which mathematical functions could be applied to extract relevant information.

This method has a significant impact on the total efficacy of the cardinal co-efficient used in the system and is the first drawback in this framework. While carrying out the computations and retrieving real time data is often automated, the formulations of the cardinal coefficient themselves would require manual inputs of experts and would play a crucial role for the accuracy of the framework. There are various challenges involved in the process such as, clear contextualization of complexity, consistency in user input, discernment of objective goals for applying the framework itself, etc. In this analysis, we will use the cardinal model suggested by Conrow (2011) to measure the overall maturity of the structures. It should be noted that the implementation of these co-efficiencies is not sufficient for the precision of this case study and needs an extensive expert-oriented formulation in conjunction with the AHP approach as suggested by Saaty (1990). However, this paradigm adopts an open layout for its implementation via separate functional parameters. This would encourage one to use their native framework of the cardinal hierarchy optimized toward the particular technologies.

To assess the maturity of the 7 NASA Technologies, we shall use the recorded transition period of each TRL as reported by Piesen et al. (1999) to attain TRL 6. The time consumed to achieve each TRL 
is a clear indication of sophistication and the implied resources to attain the said level. Piesen et al. (1999) clearly reported the time of development of 7 NASA technologies through each TRL in a consolidated form. The 7 NASA technologies are used to compare the efficacy of the proposed model against previously established methods mentioned earlier. In this study, the transition maturity time is referred to as "Consumed Time" $\left(T_{c}\right)$ and the aggregate of all maturity time as "Absolute Time" $\left(T_{t}\right)$. We believe the technology has achieved its maturity at TRL 6, this is because the acquisition of new technology often occurs when it is at least prototype ready and TRL 6 or TRL 7 (GAO, 2020). The maturation period to TRL 1 is not recorded due to the fuzzy characteristic of the process, hence it is presumed that the consumed time to TRL 1 is 0 as shown in Table 1 . The proposed approach needs more evidence to enhance its precision, for example, where the time spent is related to the absolute time of growth towards maturity of TRL 6. It is recommended that the expected growth period of each stage be compared and that the total maturity be the sum of the specific stages of maturity.

Maturity of a technology at each state of TRL is assumed to be in discrete successive stages. This means that the technology first develops to a certain amount of maturity at TRL 1 and then must attain certain level of maturity at each successive level to proceed to the next. The mathematical maturity in this study is a quantitative term depicting the completion progress of technology development at the said TRL. The maturity at each stage is zeroed at the said stage, example, if the technology completes TRL 1 and its maturity is at the value of 0.1 , the maturity of TRL 2 is calculated from 0 in the next stage and not 0.1. This is because, though the maturity is a cumulative process, the process of development is carried out in stages, and it is our intention that we monitor the progress of each stage while also observing the overall development progress.

Cardinal variables may be explicitly modulated to each other using mathematical functions. While the cardinal variables are readily applicable, we normalise these variables for uniformity in order to optimise the total performance outcome. The ratio of the time expended $\left(T_{c}\right)$ to the cumulative time $\left(T_{t}\right)$ of the preceding stage is determined (1).

$$
T_{i}=\frac{T_{c}}{T_{t}}
$$

The cardinal coefficients of the AHP attributed TRL as proposed by Conrow (2011) is denoted as "TR $L_{i}$ " of the corresponding " $i$ " level of readiness. To study the effects of linear quantities such as time and cost we multiply the factors in accordance with traditional MCDM methods such as the WASPAS method (Chakraborty, 2015). The WTRL of the system is therefore calculated by (2).

$$
W T R L=\sum_{i=1}^{9}\left(T R L_{i} \times T_{i} \times C F_{i}\right)
$$

Where,

" $C F$ " is a linear quantitative cardinal factor influencing the technology's development at the said stage of development.

At each step, the maximum maturity value is the corresponding cardinal TRL. Due to the normalized form of the coefficients, the maximum maturity is 1 . However, in this study it may not be true due to the assumptions made and we only obtain an overall scheme of development. For example, to determine the maturity of Carbon 6 transitioning from TRL 3 to 4, the consumed time is 0.4 and the absolute time for development to TRL 6 is 1.9 as shown in Table 1. The Time Ratio is determined to be $0.4 / 1.9$. Since there are no sub criteria in the same level of Technology which may influence this maturity any further, we then multiply it to the $T R L_{i}$ of the end state, i.e. $T R L_{i=4}=0.04$. Therefore, the maturity or readiness of the technology when affected while transitioning to TRL 4 is 0.008 as shown in Table 2. This factor is a relative measure to the total amount of time spent over developing the technology. 
Table 1: TRL transition time of 7 NASA technologies Piesen et al (1999).

\begin{tabular}{|l|l|l|l|l|l|l|l|l|}
\hline $\begin{array}{l}\text { (Time } \\
\text { in } \\
\text { Years) }\end{array}$ & $\begin{array}{l}\text { Carbon } \\
6 \\
\text { Thermal } \\
\text { Barrier }\end{array}$ & $\begin{array}{l}\text { Fibre } \\
\text { Preform } \\
\text { Seal }\end{array}$ & $\begin{array}{l}\text { Non- } \\
\text { Destructive } \\
\text { Evaluation }\end{array}$ & $\begin{array}{l}\text { Tailless } \\
\text { Fighter }\end{array}$ & $\begin{array}{l}\text { Thrust } \\
\text { Vectoring } \\
\text { Nozzle }\end{array}$ & $\begin{array}{l}\text { Low } \\
\text { Emission } \\
\text { Combustion }\end{array}$ & $\begin{array}{l}\text { Direct } \\
\text { To }\end{array}$ \\
\hline$T_{c 1}$ & 0 to 1 & 0 & 0 & 0 & 0 & 0 & 0 & 0 \\
\hline$T_{c 2}$ & 1 to 2 & 0.4 & 1 & 0.5 & 3 & 0.3 & 1 & 0.2 \\
\hline$T_{c 3}$ & 2 to 3 & 0.4 & 1.5 & 1 & 1 & 0.3 & 1 & 0.1 \\
\hline$T_{c 4}$ & 3 to 4 & 0.4 & 1.5 & 1 & 1 & 0.4 & 1 & 0.1 \\
\hline$T_{c 5}$ & 4 to 5 & 0.5 & 1.5 & 1 & 1 & 2 & 2 & 1.1 \\
\hline$T_{c 6}$ & 5 to 6 & 0.2 & 6 & 1 & 2 & 2 & 4 & 0.1 \\
\hline$T_{t}$ & 1 to 6 & 1.9 & 11.5 & 4.5 & 8 & 5 & 9 & 1.6 \\
\hline
\end{tabular}

\section{RESULTS AND DISCUSSIONS}

As seen in Table 2, we can now clearly determine the amount of time spent on the basis of the cardinal coefficient assigned to each stage of growth. i.e., the conviction of the cardinal coefficient first assimilates the associated complexity of technological growth using subjective influences and professional judgement. When paired with the time dimension, we observe the trend of time distribution and thus, potential resource consumption based on the allocated development time for each TRL for current or potential development ventures. It is clear that the "Fibre Preform seal" used the greatest amount of time to hit TRL 6 from TRL 5 and the "Direct to" least. Here the cardinal coefficients are uniform for all 7 technologies as the $T R L_{i}$ are used from the model described by (Conrow, 2011) and thus the complexity of each TRL is comparable. In the same manner, the allocation of assets in an organisation may be conveniently tracked.

Industries now use advanced project management systems which effectively track the daily and longterm progress of multidisciplinary teams. However, these tracking functions do not consider the complexity of the technology while computing the resource and time consumption. The cardinal coefficients while taking into consideration of the said complexities easily demarcates the global weights to each segment of development and normalizes the trend. The method presented in this paper can be applied as a module in a larger system monitoring tool or may be employed as a small-scale testing criterion individually. The framework enables quick readability and monitoring of the time allocation and schedule slippage while considering the complexity of each individual technology, or by creating a uniform scale of complexity for each development stage. The example of the latter is shown in Figure 1 while also indicating a model of schedule slippage function added to the chart.

Table 2: Maturity of 7 NASA technologies using the WTRL method.

\begin{tabular}{|l|l|l|l|l|l|l|l|}
\hline TRL & 0 to 1 & 1 to 2 & 2 to 3 & 3 to 4 & 4 to 5 & 5 to 6 & \multirow{2}{*}{ Total } \\
\hline TRLi & 0.01 & 0.02 & 0.03 & 0.04 & 0.07 & 0.1 & \\
\hline Carbon 6 Thermal Barrier & 0 & 0.004 & 0.006 & 0.008 & 0.018 & 0.011 & 0.047 \\
\hline Fibre Preform Seal & 0 & 0.002 & 0.004 & 0.005 & 0.009 & 0.052 & 0.072 \\
\hline Non-Destructive Evaluation & 0 & 0.002 & 0.007 & 0.009 & 0.016 & 0.022 & 0.056 \\
\hline Tailless Fighter & 0 & 0.008 & 0.004 & 0.005 & 0.009 & 0.025 & 0.051 \\
\hline Thrust Vectoring Nozzle & 0 & 0.001 & 0.002 & 0.003 & 0.028 & 0.040 & 0.074 \\
\hline Low Emission Combustion & 0 & 0.002 & 0.003 & 0.004 & 0.016 & 0.044 & 0.069 \\
\hline Direct To & 0 & 0.003 & 0.002 & 0.003 & 0.048 & 0.006 & 0.062 \\
\hline
\end{tabular}




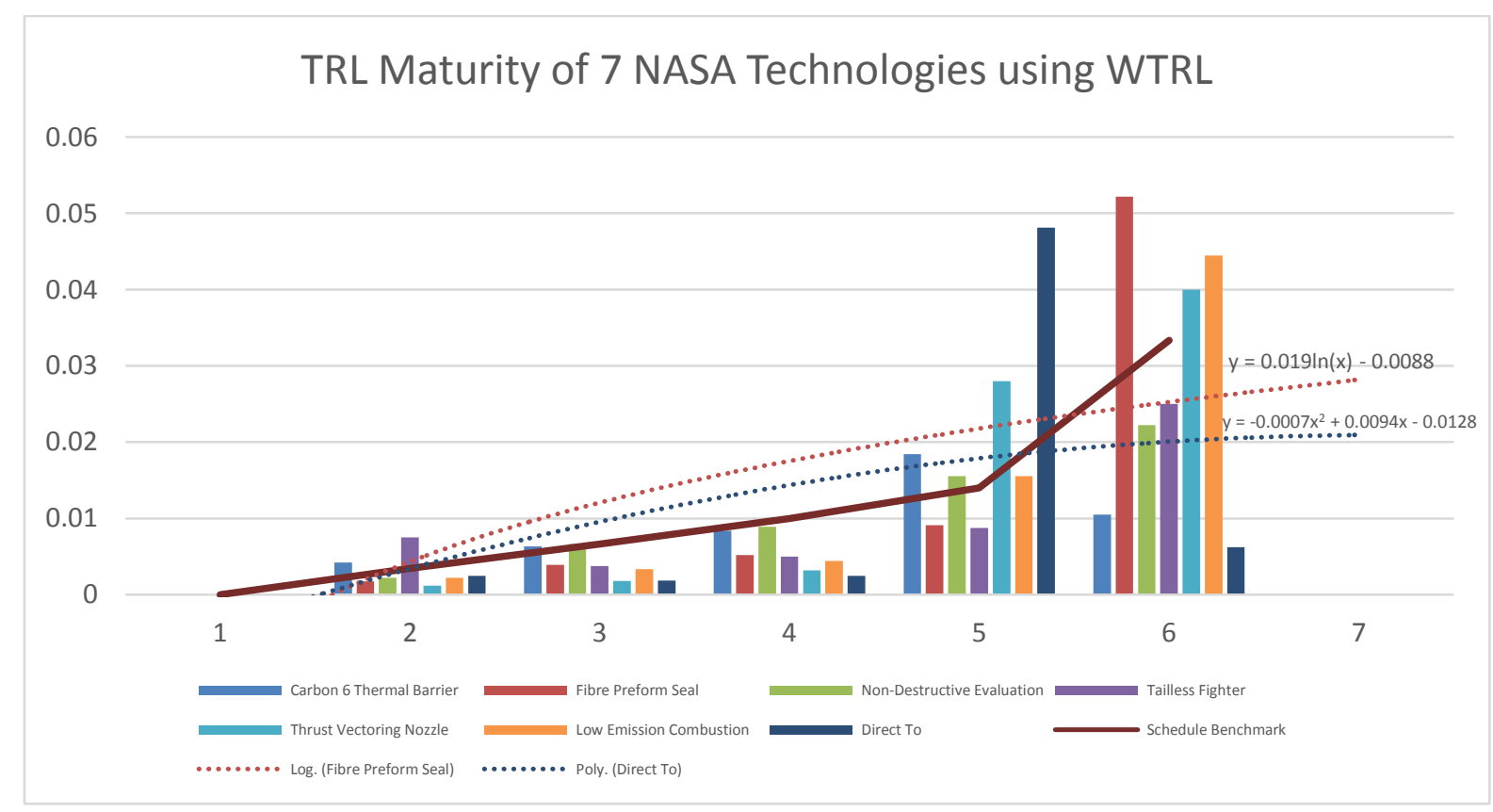

Figure 1. TRL maturity of 7 nasa technologies with forecast and schedule benchmarking using WTRL.

The approach makes it possible for other strategic initiatives to develop around it. A trend line may be drawn through the whole project to quickly assess the timing of slippage and construction threats. In this case, the placement of the trend line would be the product of the desired time and the cardinal coefficient divided by the absolute time as shown in (3). The absolute time is used to normalize the data to compare against the computed data.

$$
\text { Benchmark }=\frac{\text { No. of years } \times T R L_{i}}{T_{t}}
$$

The benchmark for each TRL may be further adjusted to attain a higher degree of control as seen in Table 3. In addition, forecasting models may be used to predict the amount of capital that will be required to further evolve the technologies. Forecast functions can be added to the interpreted data for predicting the resources which may be consumed in the proceeding phase of development. Two such functions are seen in Figure 1 (dotted lines) indicating the resource distribution criteria for Fibre Preform Seal and Direct To technologies. The forecast line is created using the Microsoft Excel chart function, which use the logarithmic and polynomial curve fitting functions to determine the forecast. Advanced automated methods can be employed based on the trend witnessed.

While the system suggested by Fahimian and Behdinan (2015) used the cardinal coefficient to determine the maturity of the technology using the AHP equation, the TRL was progressive and assumed to be the aggregate function of time to compensate for its complexity. According to the mathematical approach the accuracy of the matrix is often assumed to be reasonable. The consistency ratio as originally defined by Saaty (1990) indicates that there are no human errors conducting pairwise comparisons and that the conceptual credibility of the scores is preserved. Related conclusions as suggested by the former can be obtained using aggregate time maturity and normalizing the available data as seen in Figure 2. It was found that the method struggled to yield significant results when assimilating the cardinal coefficients. The WTRL Method proposed in this study clearly demarcated the trend of individual technologies using the AHP attributed TRL coefficients as shown in Figure 3. 
Table 3: Schedule benchmarking of TRL to monitor slippage.

\begin{tabular}{|l|l|l|l|l|l|l|}
\hline TRL & 0 to 1 & 1 to 2 & 2 to 3 & 3 to 4 & 4 to 5 & 5 to 6 \\
\hline $\begin{array}{l}\text { Benchmarking Time } \\
\text { in Years) }\end{array}$ & 0 & 2 & 1 & 2 & 1 & 3 \\
\hline Cardinal Value & 0 & 0.004 & 0.007 & 0.01 & 0.014 & 0.033 \\
\hline
\end{tabular}

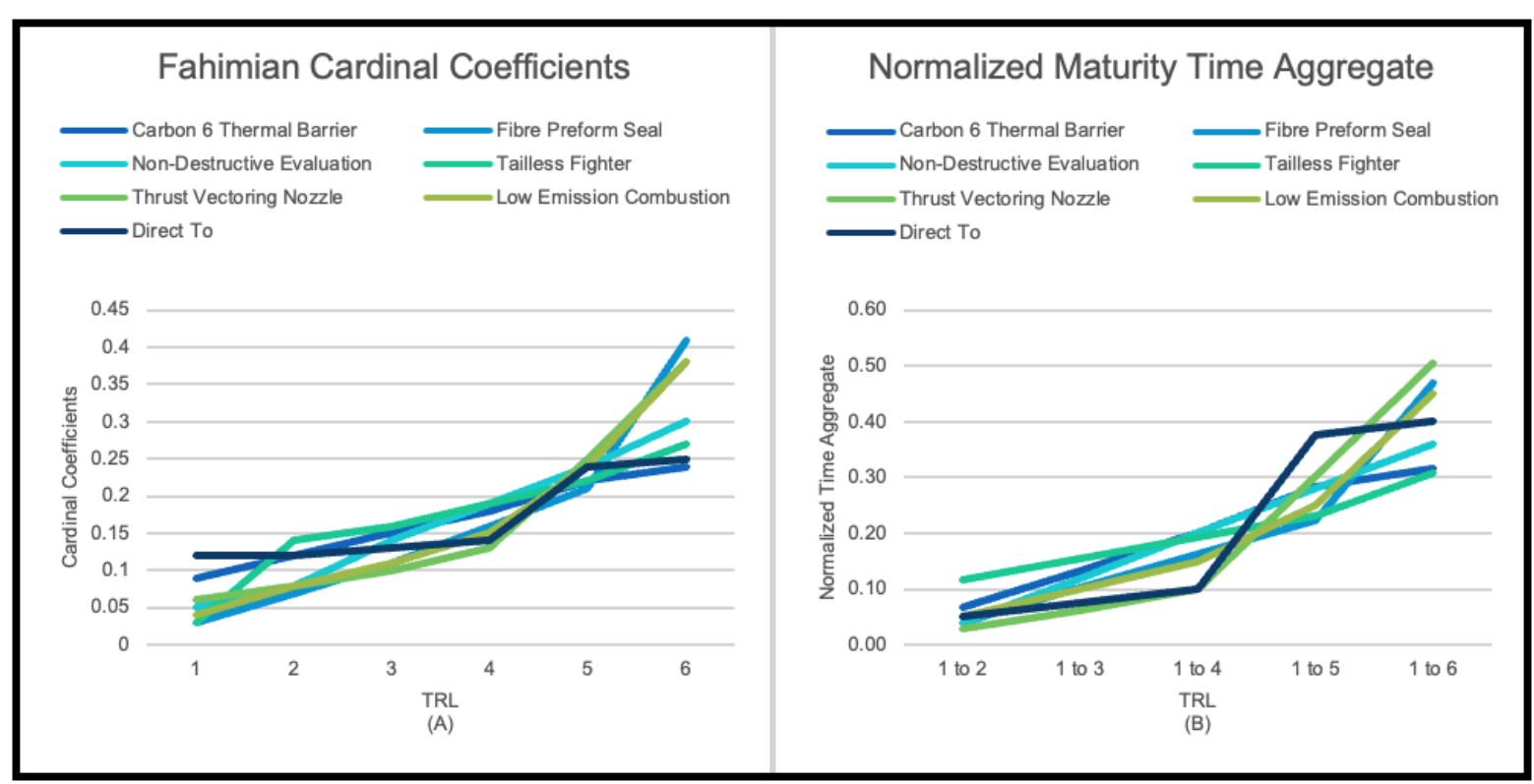

Figure 2. A: Cardinal coefficients calculated by Fahimian et al. B: Normalized aggregated sum of maturity time.

WTRL Cardinal Coefficients

0.06

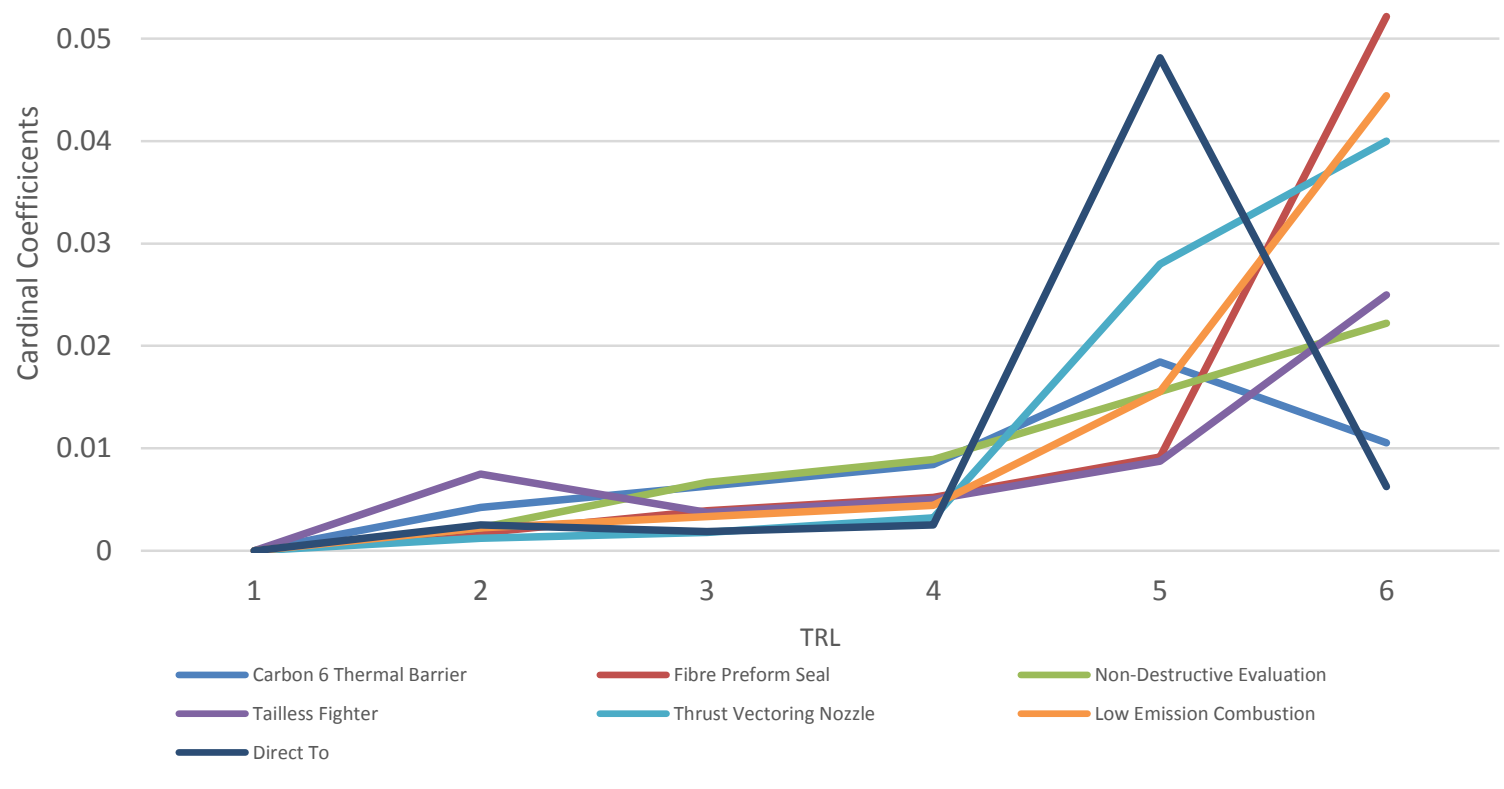

Figure 3. WTRL cardinal coefficient. 


\section{CONCLUSIONS}

Compared to previous models as suggested by various authors, the proposed framework utilizes an open cardinal model to determine technology maturity. The structure uses a simplistic weight-factor methodology that enacts ease of usability as once the framework is set up it can readily accept inputs and automate data. In this study the maturity trend of the system is determined using the elapsed transition time of the projects through TRL 1 to 6 . It is observed to be effective for tracking schedule slippage at individual TRL. To expand on the dimensions of the maturity assessment we can further multiply cardinal quantities such as cost and quality factors to determine the overall expenditure of resources towards each technology. The model aims towards the clear demarcation of risk factors associated with various parameters of technology development. It overcomes many limitations such as eliminating minimum time allocation for computation, repeated need for pairwise comparisons. It uses a flexible cardinal framework which can readily interchange the cardinal coefficients as compared to the previous framework suggested by Fahimian et al. (2015). It identifies clear and consistent entry and exit points of the maturity assessment process. The framework continues to have numerous restrictions towards its application such as integrating complexities of sub-processes in the development process, efficient methods to eliminate pairwise comparisons and user inputs to compute complexity. The framework presented in this study uses a linear model which may significantly reduce the computational time for a large number of technologies and stage processes. The model can be further expended towards other stage gate development processes with models similar to that of TRL.

\section{ACKNOWLEDGMENTS}

We would like to acknowledge the support of the Natural Sciences and Engineering Research Council of Canada (NSERC), [NSERC Alliance Grants \# ALLRP 550058 - 20].

\section{REFERENCES}

Asst. Secretary of Defense for Research and Engineering (2011), Technology Readiness Assessment (TRA) Guidance, Department of Defense, Washington, D.C.

Azizian, N., Shahram, S. and Mazzuchi, T. (2009), "A Comprehensive Review and Analysis of Maturity Assessment Approaches for Improved Decision Support to Achieve Efficient Defense Acquisition", Proceedings of the World Congress on Engineering and Computer Science 2009, Vol II, San Francisco, USA.

Behdinan, K. (2020), “Design Readiness Level (DRL) and Design Axis Principles”, Workshop Lecture, Transdisciplinary and Cross-cultural Learning, Society for Design Practise and Process Science.

Blanke, J. (2010), Technology Readiness Levels Demystified. [online] NASA. Available at: <https://www.nasa.gov/topics/aeronautics/features/trl_demystified.html> [Accessed 17 November 2020].

Chakraborty, S., Zavadskas, E., Antucheviciene, J. (2015), “Applications of WASPAS method as a multi-criteria decision-making tool", Economic computation and economic cybernetics studies and research, Academy of Economic Studies, Vol 49, pp 5-22.

Conrow, E., (2011), "Estimating Technology Readiness Level Coefficients", Journal of Spacecraft and Rockets, vol. 48, no. 1, pp. 146-152. https://dx.doi.org/10.2514/1.46753.

Cornford, S. and Sarsfield, L. (2004), "Quantitative Methods for maturing and Infusing Advanced Spacecraft Technology”, IEEE Aerospace Conference Proceedings, pp. 663-681.

Dubos, G.F., Saleh, J.H. and Braun, R. (2008), "Technology Readiness Level, Schedule Risk, and Slippage in Spacecraft Design”, Journal of Spacecraft and Rockets, vol. 45(4), pp.836-842. https://dx.doi.org/10.2514/1.34947

Fahimian, M. and Behdinan, K. (2017), "On characterization of technology readiness level coefficients for design”, DS 87-2 Proceedings of the 21st International Conference on Engineering Design (ICED 17), vol. 2, pp. 309-316.

GAO (2020), Technology Readiness Assessment Guide - Best Practices for Evaluating the Readiness of Technology for Use in Acquisition Programs and Projects, U.S. Government Accountability Office, vol. GAO-20-48G.

GAO (2008), Better Weapon Program Outcomes Require Discipline, Accountability, and Fundamental Changes in the Acquisition Environment, U.S. Government Accountability Office, vol. GAO-08-782 T, GAO, Ed, 2008.

GAO (1999), Best Practices: Better Management of Technology Development Can Improve Weapon System Outcomes, U.S. Government Accountability Office.

Lee, T. and Thomas, L.D. (2003), "Cost Growth Models for NASA 'S Programs : A Summary”, Journal of probability and Statistical Science, vol. 1(2), pp.265-279. 
Magnaye, R., Sauser, B. and Ramirez-Marquez, J. (2010), "System development Planning Using Readiness Levels in a Cost of Development Minimization Model”, Systems Engineering, vol. 13(4), pp.311-323. https://dx.doi.org/10.1002/sys.20151

Mankins, J.C. (1995), “Technology Readiness Levels”, NASA office of Space and Technology, White Paper, p.5.

Mankins, J. C. (2009), "Technology readiness and risk assessments: A new approach”. Acta Astronautica, vol. 65(9-10), pp.1208-1215. https://dx.doi.org/10.1016/j.actaastro.2009.03.059

Mihaly, Heder (2017), "From NASA to EU: the evolution of the TRL scale in Public Sector Innovation", The Innovation Journal. Vol 22: pp 1-23. [online] Way back machine. Available at: https://web.archive.org/web/20171011071816/https:/www.innovation.cc/discussionpapers/22_2_3_heder_nasa-to-eu-trl-scale.pdf [Accessed 17 November 2020].

Olechowski, A., Eppinger, S., Joglekar, N. and Tomaschek, K. (2020), "Technology readiness levels: Shortcomings and improvement opportunities”, Systems Engineering, vol. 23, no. 4, pp. 395-408. https://dx.doi.org/10.1002/sys.21533.

Peisen, D.J., Schulz, C.R., Golaszewki, R.S., Ballard, B.D. and Smith, J. (1999), Time Required to Mature Aeronautic Technologies to Operational Readiness, SAIC Report.

S. Revfi, J. Wilwer, K. Behdinan and A. Albers (2020), "Design Readiness of Multi-Material Concepts: Manufacturing And Joining Technology Integrated Evaluation Of Concept Maturity Levels Using Cardinal Coefficients", Proceedings of the Design Society: DESIGN Conference, vol. 1, pp. 1067-1076.

Saaty, T. (1990), "How to make a decision: The analytic hierarchy process", European Journal of Operational Research, vol. 48, no. 1, pp. 9-26. https://dx.doi.org/10.1016/0377-2217(90)90057-i.

U.S. Department of Energy, (2011), Technology Readiness Assessment Guide, DOE G 413.3-4A, Washington, D.C.

Valerdi, R. and Kohl, R.J. (2004), “An approach to technology risk management”, Engineering Systems Division Symposium, MIT, Cambridge, MA. 


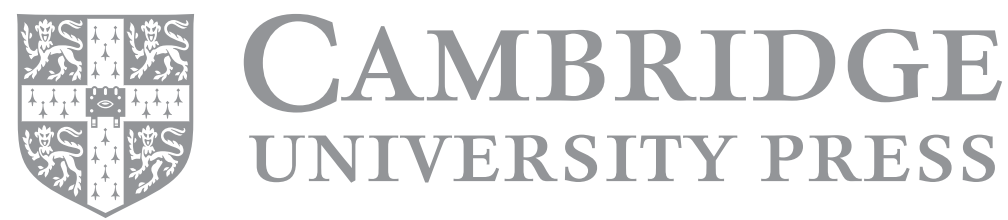

